\title{
Morphological Classification of the Yeast Vacuolar Protein Sorting Mutants: Evidence for a Prevacuolar Compartment in Class E vps Mutants
}

\author{
Christopher K. Raymond,* Isabelle Howald-Stevenson, \\ Carol A. Vater, $†$ and Tom H. Stevens
}

Institute of Molecular Biology, University of Oregon, Eugene, Oregon 97403

Submitted August 13, 1992; Accepted October 5, 1992

\begin{abstract}
The collection of vacuolar protein sorting mutants (vps mutants) in Saccharomyces cerevisiae comprises of 41 complementation groups. The vacuoles in these mutant strains were examined using immunofluorescence microscopy. Most of the vps mutants were found to possess vacuolar morphologies that differed significantly from wild-type vacuoles. Furthermore, mutants representing independent vps complementation groups were found to share aberrant morphological features. Six distinct classes of vacuolar morphology were observed. Mutants from eight vps complementation groups were defective both for vacuolar segregation from mother cells into developing buds and for acidification of the vacuole. Another group of mutants, represented by 13 complementation groups, accumulated a novel organelle distinct from the vacuole that contained a late-Golgi protein, active vacuolar $\mathrm{H}^{+}$-ATPase complex, and soluble vacuolar hydrolases. We suggest that this organelle may represent an exaggerated endosome-like compartment. None of the vps mutants appeared to mislocalize significant amounts of the vacuolar membrane protein alkaline phosphatase. Quantitative immunoprecipitations of the soluble vacuolar hydrolase carboxypeptidase $Y$ (CPY) were performed to determine the extent of the sorting defect in each vps mutant. A good correlation between morphological phenotype and the extent of the CPY sorting defect was observed.
\end{abstract}

\section{INTRODUCTION}

Organelle biogenesis in eukaryotic cells requires the selective assembly of specific proteins, lipids, and other constituents from a common pool of biosynthetic intermediates. The biogenesis of the yeast vacuole was chosen as a model system for organelle assembly because most vacuolar functions are dispensible for the growth of vegetative cells, and hence it was possible to generate a large collection of mutants with defects in vacuole assembly (reviewed in Klionsky et al., 1990; Raymond et al., 1992a). Most soluble and integral membrane proteins of the yeast vacuole studied to date enter the endoplasmic reticulum and traverse the secretory pathway through the Golgi complex (Stevens

\footnotetext{
* Present Address: ZymoGenetics, Inc., 4225 Roosevelt Way NE, Seattle, WA 98115.

$\dagger$ Present address: Immunogen, Inc., 148 Sydney Street, Cambridge, MA 02139-4239.
}

et al., 1982; Klionsky et al., 1988; Klionsky and Emr, 1989; Moehle et al., 1989; Roberts et al., 1989; Hirsh et al., 1991). Vacuolar proteins appear to be sorted from cell surface and resident Golgi proteins in a late Golgi compartment. This was elegantly documented in the case of the soluble vacuolar glycoprotein carboxypeptidase Y (CPY), which was shown to be sorted to the vacuole after passage through the most distal Golgi compartment characterized in Saccharomyces cerevisiae (Graham and Emr, 1991).

The precursor form of CPY carries positive sorting information that directs the protein to the vacuole (Johnson et al., 1987; Valls et al., 1987, 1990). In the absence of this sorting signal, the protein is secreted (Stevens et al., 1986). To identify genes whose products participate in the sorting process, positive selections for mutants that secrete CPY were devised (Bankaitis et al., 1986; Rothman and Stevens, 1986). Complementation testing among a large collection of such vacuolar protein sorting (vps) mutants has indicated that as many as 40 
VPS genes influence CPY sorting (Robinson et al., 1988; Rothman et al., 1989a). Additional analyses have shown that all of the vps mutants exhibit defects in the sorting of several other soluble vacuolar proteins (Robinson et al., 1988; Rothman and Stevens, 1986). The large number of vps complementation groups demonstrates that vacuole assembly is a complex process.

Phenotypic characterization of the vps mutants suggests that a wide range of vacuolar biogenesis defects may influence the fidelity of soluble protein sorting. Certain mutants were found to have gross alterations in vacuolar morphology or to lack an acidic vacuolar lumen (Rothman and Stevens, 1986; Banta et al., 1988; Rothman et al., 1989a,b). Initial characterization of vacuolar morphology in the vps mutant collection was conducted at the level of the light microscope using the vacuolar lumenal marker fluorescein isothiocyanate (FITC) and Nomarski optics, as well as by thin-section electron microscopy (Banta et al., 1988). These techniques were sufficient to divide the vps mutant collection into three classes based on vacuolar morphology (Banta et al., 1988). Most of the vps mutants, class A, contained vacuoles that appeared similar to those in wild-type cells. Certain mutants, class B, contained numerous small vacuole-like compartments. Four vps mutants, class $C$, exhibited the most extreme defect in vacuolar morphology and appeared to lack vacuoles altogether.

Although initial studies indicated that the vast majority of vps strains possessed relatively normal vacuoles (Banta et al., 1988; Rothman et al., 1989a), recent advances in fluorescence microscopy and the availability of antibodies that recognize vacuolar membrane proteins have provided powerful new tools for phenotypic analysis (Pringle et al., 1989; Roberts et al., 1991). These techniques provided a clear demonstration that vacuole inheritance in wild-type cells occurs via vacuolar segregation structures; the term "vacuole inheritance" refers to the process by which daughter cells receive vacuolar material from mother cells (Zubenko et al., 1982; Weisman et al., 1987), and the term vacuolar "segregation structure" refers to the morphologically distinct projection of mother cell vacuoles into developing buds during a specific period in bud enlargement (Weisman and Wickner, 1988; Raymond et al., 1990; Weisman et al., 1990; Gomes de Mesquita et al., 1991; Shaw and Wickner, 1991). Microscopic analysis demonstrated that vps3 and vps34 mutants were defective for vacuolar segregation (Herman and Emr, 1990; Raymond et al., 1990). The fact that both mutants were originally thought to have wild-type vacuoles prompted us to reexamine the vps mutant collection using indirect immunofluorescence microscopy. We report that the vacuoles in most of the vps mutants differ significantly from the wild-type organelle and that the entire collection of vps mutants can be subdivided into specific groups based on the morphological traits of their vacuoles.

\section{MATERIALS AND METHODS}

\section{Strains, Media, Materials, and Antibodies}

The yeast strains used in this study are listed in Table 1 . New vacuolar protein localization $(v p l)$ complementation groups were identified by selection for mutants $\left(\mathrm{Vps}^{-}\right)$that secrete the vacuolar protein $\mathrm{CPY}$ followed by complementation testing as described previously (Rothman and Stevens, 1986; Rothman et al., 1989a), except that CPY secretion was monitored using a plate overlay immunoblotting technique (Rothman et al., 1986; Roberts et al., 1991). The vps27- 1 :: LEU2 mutation was constructed by substituting the LEU2 gene for a portion of the VPS27 open reading frame and by transplacement of this construct into the indicated yeast strains. DNA was transformed into yeast strains by electroporation using a Bio-Rad (Richmond, CA) gene pulser as recommended by the manufacturer. Rich growth medium was yeast peptone dextrose (YPD), minimal medium was synthetic dextrose (SD) supplemented with appropriate nutrients, and sporulation medium was a standard recipe, all as described in Sherman et al. (1982). The Escherichia coli strain used in this study was MC1061 (Casadaban and Cohen, 1980).

Antibodies that recognize alkaline phosphatase (ALP), the product of the PHO8 gene, were generated by subcloning the $\sim 1000$ base pair (bp)Xba I - Sal I fragment of PHO8 (Kaneko et al., 1987) into the E. coli protein expression vector pEXP2 (Raymond et al., 1990). The $38-\mathrm{kDa}$ ALP protein fragment produced from this plasmid, PGP100, was purified from $E$. coli and injected into rabbits as described in Roberts et al. (1989). A second overlapping $\sim 875$ bp Bgl II - Sal I fragment from the $\mathrm{PHO} 8$ gene was also subcloned into pEXP2 to give pGP102, and the resulting $35-\mathrm{kDa}$ protein fragment was used to construct an affinity column by linking it to cyanogen bromide-activated Sepharose (Sigma Chemical, St Louis, MO). ALP-specific antibodies were affinity purified from rabbit serum as described by Raymond $e t$ al. (1990), and microscopy-grade adsorbed antibodies that recognize ALP were prepared as described by Roberts et al. (1991). Monoclonal antibodies that recognize the $60-\mathrm{kDa}$ subunit of the vacuolar $\mathrm{H}^{+}$adenosine triphosphatase (ATPase) (V-ATPase) were prepared by Kane et al. $(1989,1992)$. For immunofluorescence, rabbit antibodies directed against yeast CPY were affinity-purified using a CPY-Sepharose column, as described above. Polyclonal rabbit anti-CPY serum used for immunoprecipitations was prepared by subcloning the 1150 bp Bam HI fragment from the CPY structural gene, PRC1 (Stevens et al., 1986), into the $E$. coli protein expression vector pEXP2 (Raymond et al., 1990). The $42-\mathrm{kDa} C P Y$ fragment was purified from $E$. coli extracts and injected into rabbits as described by Roberts et al. (1989). Secondary antibodies for indirect immunofluorescence were purchased from Jackson ImmunoResearch (West Grove, PA) and prepared according to the recommendations of the manufacturer. Other reagents were from Sigma Chemical, except as specified below.

\section{Fluorescence Microscopy}

Vacuolar morphology was examined in fixed yeast strains by indirect immunofluorescence techniques essentially as described by Roberts et al. (1991). Ten-milliliter log phase cultures at a density of 2.5-5 $\times 10^{6}$ cells $/ \mathrm{ml}$ in YPD at $30^{\circ} \mathrm{C}$ were fixed by the addition of $1.2 \mathrm{ml}$ $37 \%$ formaldehyde (J. T. Baker Chemical, Phillipsburg, NJ) with gentle shaking. After $30 \mathrm{~min}$, the cells were transferred to $2 \mathrm{ml}$ of standard fixative (Roberts et al., 1991) and shaken overnight (12-16 h) at room temperature. The cells were then harvested in a clinical centrifuge, resuspended in $1 \mathrm{ml}$ of $200 \mathrm{mM}$ tris(hydroxymethyl)aminomethane (Tris), pH 8.0, $20 \mathrm{mM}$ EDTA, and $1 \% \beta$-mercaptoethanol, transferred to a microfuge tube, and incubated for $10 \mathrm{~min}$. Pelleted cells were resuspended in spheroplast buffer containing $1.2 \mathrm{M}$ sorbitol, $50 \mathrm{mM}$ $\mathrm{KPO}_{4}, \mathrm{pH} 7.3,1 \mathrm{mM} \mathrm{MgCl} 2,15 \mu \mathrm{g} / \mathrm{ml}$ zymolyase $100 \mathrm{~T}$ (ICN Biomedicals, Irvine, CA), and $25 \mu \mathrm{l} / \mathrm{ml}$ glusulase (DuPont, Boston, MA) and incubated with occasional shaking for $30 \mathrm{~min}$ at $30^{\circ} \mathrm{C}$. Spheroplasted cells were harvested by centrifugation at $\sim 5000 \times g$ for $30 \mathrm{~s}$, washed once in $1 \mathrm{ml}$ of $1.2 \mathrm{M}$ sorbitol, and split into two $500 \mu \mathrm{l}$ aliquots. One aliquot was treated with $5 \%$ sodium dodecyl sulfate (SDS) for $5 \mathrm{~min}$ for ALP staining (by adding $500 \mu \mathrm{l} 10 \%$ SDS in $1.2 \mathrm{M}$ sorbitol), and 
the other was treated with $1 \%$ SDS for $30 \mathrm{~s}$ for $60-\mathrm{kDa}$ V-ATPase subunit staining. Both treated aliquots were subsequently washed twice with $1 \mathrm{ml}$ of $1.2 \mathrm{M}$ sorbitol and resuspended in $1 \mathrm{ml} 1.2 \mathrm{M}$ sorbitol.

Forty-microliter aliquots of fixed treated cells were placed on polylysine-coated multiwell test slides (ICN Biomedicals) that were prepared by placing a $1 \mathrm{mg} / \mathrm{ml}$ solution of polylysine bromide (Sigma Chemical) into each well for $30 \mathrm{~s}$ followed by six washes with water and air drying. After $10 \mathrm{~min}$, the wells were washed three times with phosphate-buffered saline containing $5 \mathrm{mg} / \mathrm{ml}$ bovine serum albumin (PBS-BSA) (Roberts et al., 1991), and the slides were incubated in a humid chamber for $15 \mathrm{~min}$. Antibodies were then added. For ALP staining, the primary antibody was used at a 1:10 dilution, and the secondary antibodies were used at a 1:100 dilution of biotinylated goat anti-rabbit followed by a 1:100 dilution of FITC-conjugated streptavidin. For $60-\mathrm{kDa}$ V-ATPase subunit staining, the primary antibody was used at a 1:20 dilution, and the secondary antibodies were used at a 1:200 dilution of biotinylated goat anti-mouse followed by a 1:200 dilution of FITC-streptavidin. For the ALP/60-kDa doublelabeling experiment (Figure 7), 1:10 ALP and 1:10 60-kDa V-ATPase subunit antibodies were added in the first incubation, followed by 1 : 100 biotinylated goat anti-rabbit and 1:100 rhodamine-conjugated goat anti-mouse in the second incubation and 1:100 FITC-streptavidin in the final incubation. For the CPY $/ 60-\mathrm{kDa}$ subunit double-labeling experiment (Figure 7), 1:1000 CPY and 1:10 60-kDa V-ATPase subunit antibodies were added in the first incubation, followed by 1:100 FITCgoat anti-rabbit and 1:200 biotinylated goat anti-mouse in the second incubation, and 1:200 rhodamine-streptavidin in the final incubation. The concentration of primary antibody solutions was not determined and the dilutions used for optimal staining were determined empirically. Secondary antibodies were supplied or prepared as $1-\mathrm{mg} / \mathrm{m}$ solutions. All antibody incubations were for $1 \mathrm{~h}$, and several PBS BSA washes were conducted between antibodies. After antibody labeling was complete and the wells were washed several times mounting medium (Pringle et al., 1989) was added to each well, and the slides were covered with a coverslip. The cells were viewed and photographed as described by Raymond et al. (1990).

Quinacrine staining of live cells was conducted as described previously (Roberts et al., 1991).

\section{CPY Immunoprecipitation}

Yeast cultures were grown overnight in SD-Met to an optical density at $600 \mathrm{~nm}$ of $\sim 1\left(\mathrm{OD}_{600}=1\right)$. Cells were centrifuged and resuspended at $\mathrm{OD}_{600}=1$ in SD-Met, $50 \mathrm{mM} \mathrm{KPO}$, pH 5.7, and $2 \mathrm{mg} / \mathrm{mL} \mathrm{BSA}$ The cultures were pulse-labeled for $10 \mathrm{~min}$ at $30^{\circ} \mathrm{C}$ with $200 \mu \mathrm{Ci} / 1$ $\mathrm{OD}_{600}$ of cells with $\left.\left[^{35} \mathrm{~S}\right] \mathrm{MET}{ }^{35} \mathrm{~S}\right] \mathrm{CYS}$ (EXPRE ${ }^{35} \mathrm{~S}^{35} \mathrm{~S}$ label, New England Nuclear, Boston, MA). Labeled cultures were chased with $80 \mu \mathrm{g} / \mathrm{ml}$ of Met and Cys at $30^{\circ} \mathrm{C}$ for $30 \mathrm{~min}$. The pulse-chase was terminated by the addition of $\mathrm{NaN}_{3}$ to $10 \mathrm{mM}$. The cells were spheroplasted separated into intracellular and extracellular fractions, and CPY was immunoprecipitated from each fraction as described by Stevens $e t a l$. (1986) with the following modifications. Intracellular and extracellular fractions were heated for $5 \mathrm{~min}$ at $100^{\circ} \mathrm{C}$ after the addition of 0.5 $\mathrm{mM}$ phenylmethylsulfonyl fluoride, $1 \mu \mathrm{g} / \mathrm{ml}$ pepstatin, $1 \mu \mathrm{g} / \mathrm{ml}$ leupeptin, $0.13 \%$ SDS, and $0.13 \%$ Triton $X-100$ (final concentrations) Samples were preadsorbed with $50 \mu \mathrm{l}$ of $10 \%$ IgGSORB (The Enzyme Center, Malden, MA) for $15 \mathrm{~min}$ on ice. The supernatants resulting after centrifugation were incubated with anti-CPY polyclonal antibody for $1 \mathrm{~h}$ on ice. Another aliquot of IgGSorb was added, and after $1 \mathrm{~h}$ on ice the precipitated immune complexes were washed twice with $10 \mathrm{mM}$ Tris $\mathrm{HCl}, \mathrm{pH} 8.0,0.1 \%$ SDS, $0.1 \%$ Triton $X-100$, and $2 \mathrm{mM}$ EDTA. Immunoprecipitated samples were analyzed by SDS-polyacrylamide gel electrophoresis and fluorography. The radioactivity of immunoprecipitated CPY in the intracellular and extracellular fractions was quantified using an AMBIS Radioanalytic Imaging System (Ambis Systems, San Diego, CA).

\section{RESULTS}

\section{Analysis of Vacuolar Morphology and Protein Sorting in the vps Mutant Collection}

The vps mutants, all of which were selected for secretion of CPY or a CPY fusion protein, were generated in two different laboratories and originally designated as vacuolar protein localization $(v p l)$ mutants (Rothman and Stevens, 1986; Rothman et al., 1989a) or vacuolar protein targeting (vpt) mutants (Bankaitis et al., 1986; Robinson et al., 1988). The common vps nomenclature was introduced because many of the vpl and vpt mutants defined the same complementation groups, i.e., they carried mutations in many of the same genes (Robinson et al., 1988; Rothman et al., 1989a). There have been several revisions to the vps mutant list since the last available publication (Klionsky et al., 1990), and the current compilation of vps complementation groups is shown in Table 2.

Vacuoles were visualized using indirect immunofluorescence techniques (Pringle et al., 1989; Roberts et al., 1991). Two antibody preparations that recognize either ALP or the $60-\mathrm{kDa}$ subunit of the vacuolar $\mathrm{H}^{+}$-ATPase (V-ATPase) were used. ALP is an integral membrane protein of the vacuole (Kaneko et al., 1987; Klionsky and Emr, 1989), and antibodies directed against ALP clearly delineate the vacuolar membrane (Raymond $e t$ al., 1990; Roberts et al., 1991). The 60-kDa V-ATPase subunit is a peripheral membrane protein that associates as part of a multi-subunit complex with the cytoplasmic face of the vacuole (Kane et al., 1989, 1992; Yamashiro et al., 1990). Antibodies directed against the 60-kDa VATPase subunit stain the vacuolar membrane in wildtype cells, and they yield a diffuse cytoplasmic staining pattern in mutants that fail to assemble an active VATPase complex (Kane et al., 1992). Vital staining with quinacrine was also used as a qualitative assay of vacuolar acidification in live cells. Quinacrine is a basic fluorescent compound that accumulates in acidified intracellular compartments such as the yeast vacuole; loss of vacuolar acidification due to dissipation of the $\mathrm{pH}$ gradient with $200 \mathrm{mM}$ ammonium acetate, inhibition of the V-ATPase with the drug bafilomycin $A_{1}$, or mutations that perturb V-ATPase function largely abolish quinacrine staining (Weisman et al., 1987; Banta et al., 1988; Rothman et al., 1989b; Yamashiro et al., 1990). Vacuolar protein sorting was monitored by pulse-chase labeling of cells, immunoprecipitation of CPY from intracellular and extracellular fractions, and quantitation of CPY in the immunoprecipitates.

At least two mutant strains from each complementation group were examined by the techniques described above. Additional alleles were examined for groups where the initial two mutants gave inconsistent results. The pairs of MAT $\alpha$ and MATa vps mutant alleles reported in this study are shown in Table 1 . Within the 41 vps mutant groups studied, six different classes of mutant vacuolar morphology were observed that could be readily differentiated from one another. To be consistent with previous publications, the nomenclature of Banta et al. (1988) was used and extended to include additional classes, as described in detail in later sections. 
Table 1. Yeast strains used in this study

\begin{tabular}{|c|c|c|}
\hline Strains & Genotype & Source \\
\hline SF838-9D & MAT $\alpha$, ade6, his4, leu2, ura3, pep 4 & Rothman et al., 1989a \\
\hline SF838-9DR2L1 & MATa, his4, leu2, lys2, ura3, pep4 & Rothman \\
\hline JHRY20-2C & MTAa, his3, leu2, ura3 & Rothman et al., 1986 \\
\hline SEY6210 & $M A T \alpha$, his 3, leu2, lys2, suc2, trp1, ura3 & Robinson et al., 1988 \\
\hline SEY6211 & MATa, ade2, his3, leu2, suc2, trp1, ura3 & Robinson et al., 1988 \\
\hline SF838-9D $v p s 1-\Delta 2$ & same as SF838-9D except vps $1-\Delta 2:: L E U 2$ & Rothman et al., 1990 \\
\hline JHRY20-2C vps1- $\Delta 2$ & same as JHRY20-2C except vps1- $\Delta 2:: L E U 2$ & Rothman et al., 1990 \\
\hline$v p l 2-7, v p l 2-2^{\mathrm{a}}$ & same as SF838-1D, same as SF838-9DR2L1 & Rothman and Stevens, 1986 \\
\hline vpl3-1, vpl3-2 & same as SF838-1D, same as SF838-9DR2L1 & Rothman and Stevens, 1986 \\
\hline vpl4-1, vpl4-7 & same as SF838-1D, same as SF838-9DR2L1 & Rothman and Stevens, 1986 \\
\hline vpl5-21, vpl5-8 & same as SF838-1D, same as SF838-9DR2L1 & Rothman and Stevens, 1986 \\
\hline vpl6-27, vpl6-18 & same as SF838-1D, same as SF838-9DR2L1 & Rothman and Stevens, 1986 \\
\hline vpl7-1, vpl7-2 & same as SF838-1D, same as SF838-9DR2L1 & Rothman and Stevens, 1986 \\
\hline vpl8-24, vpl8-19 & same as SF838-1D, same as SF838-9DR2L1 & Rothman and Stevens, 1986 \\
\hline vpl9-13, vpl9-2 & same as SF383-9D, same as SF383-9DR2L1 & Rothman et al., 1989a \\
\hline vpl10-7, vpl10-27 & same as SF383-9D, same as SF383-9DR2L1 & Rothman et al., 1989a \\
\hline vpl11-58, vpl11-2 & same as SF383-9D, same as SF383-9DR2L1 & Rothman et al., 1989a \\
\hline vpl12-1, vpl12-3 & same as SF383-9D, same as SF383-9DR2L1 & Rothman et al., 1989a \\
\hline vpl13-2, vpl13-11 & same as SF383-9D, same as SF383-9DR2L1 & Rothman et al., 1989a \\
\hline vpl14-6, vpl14-12 & same as SF383-9D, same as SF383-9DR2L1 & Rothman et al., 1989a \\
\hline vpl15-9, vpl15-18 & same as SF383-9D, same as SF383-9DR2L1 & Rothman et al., 1989a \\
\hline vpl16-2, vpl16-9 & same as SF383-9D, same as SF383-9DR2L1 & Rothman et al., 1989a \\
\hline vpl17-7, vpl17-2 & same as SF383-9D, same as SF383-9DR2L1 & Rothman et al., 1989a \\
\hline$v p l 18-9, v p l 18-3^{b}$ & same as SF383-9D, same as SF383-9DR2L1 & Rothman et al., 1989a \\
\hline vpl19-10, vpl19-3 & same as SF383-9D, same as SF383-9DR2L1 & Rothman et al., 1989a \\
\hline vpl20-6, vpl20-1 & same as SF383-9D, same as SF383-9DR2L1 & This study \\
\hline vpl21-13, vpl21-1 & same as SF383-9D, same as SF383-9DR2L1 & This study \\
\hline vpl22-7, vpl22-1 ${ }^{\mathrm{b}}$ & same as SF383-9D, same as SF383-9DR2L1 & This study \\
\hline$v p l 23-5, v p l 23-2$ & same as SF383-9D, same as SF383-9DR2L1 & This study \\
\hline vpl24-4, vpl24-1 & same as SF383-9D, same as SF383-9DR2L1 & This study \\
\hline vpl25-6, vpl25-1 & same as SF383-9D, same as SF383-9DR2L1 & This study \\
\hline vpl26-2, vpl26-1 & same as SF383-9D, same as SF383-9DR2L1 & This study \\
\hline vpl27-3, vpl27-1 & same as SF383-9D, same as SF383-9DR2L1 & This study \\
\hline vpl28-4, vpl28-1 & same as SF383-9D, same as SF383-9DR2L1 & This study \\
\hline vpl30-2, vps30-1 & same as SF383-9D, same as SF383-9DR2L1 & This study \\
\hline vpl31-2, vpl31-1 & same as SF383-9D, same as SF383-9DR2L1 & This study \\
\hline CKRY2-8A & $\begin{array}{l}\text { MTAa, ade6, (his3 and/or his4), leu2, ura3, } \\
\text { pep4, pho8- } \Delta 1:: \text { LEU2, vps } 27-\Delta 1:: L E U 2\end{array}$ & This study \\
\hline$v p t 1, v p t 1^{\mathrm{d}}$ & same as SEY6210, same as SEY6211 & Robinson et al., 1988 \\
\hline vpt2, vpt2 & same as SEY6210, same as SEY6211 & Robinson et al., 1988 \\
\hline vpt3, vpt3 & same as SEY6210, same as SEY6211 & Robinson et al., 1988 \\
\hline vpt4, vpt4 & same as SEY6210, same as SEY6211 & Robinson et al., 1988 \\
\hline vpt5, vpt5 & same as SEY6210, same as SEY6211 & Robinson et al., 1988 \\
\hline vpt6, vpt6 & same as SEY6210, same as SEY6211 & Robinson et al., 1988 \\
\hline vpt7, vpt7 & same as SEY6210, same as SEY6211 & Robinson et al., 1988 \\
\hline vpt16 & same as SEY6210, same as SEY6211 & Robinson et al., 1988 \\
\hline$v p t 18$ & same as SEY6210, same as SEY6211 & Robinson et al., 1988 \\
\hline vpt 21, vpt 21 & same as SEY6210, same as SEY6211 & Robinson et al., 1988 \\
\hline vpt 30, vpt 30 & same as SEY6210, same as SEY6211 & Robinson et al., 1988 \\
\hline vpt31, vpt31 & same as SEY6210, same as SEY6211 & Robinson et al., 1988 \\
\hline
\end{tabular}

a Pairs of $v p l$ mutants used in this study are listed by allele number, with the MAT $\alpha$ mutant followed by the MATa mutant.

${ }^{\mathrm{b}}$ vpl18 and vpl22 mutants were found to define a single complementation group that was designated vpl18; all four alleles were examined in this study.

' CRY2-8A is the outcrossed haploid progeny of a diploid made by crossing JHRY20-2C pho8- $\Delta 1$ (Raymond et al., 1990) X SF838-9D vps27- $\Delta 1$. The diploid was sporulated and haploid progeny that were both pho8 $\Delta$ and vps27D were used for further study. Because the parents of the diploid strain were both His $^{-}$, but at two different HIS loci (his3 or his4), it is known that CRY2-8A is His $^{-}$, but the genotypes of the HIS loci have not been established.

d Pairs of vpt mutants used in this study are listed by allele number, with the MAT $\alpha$ mutant followed by the MATa mutant. Although all of vpt mutants were examined, only those vpt strains that do not overlap genetically with vpl mutants are shown. 
Table 2. The vpt and $v p l$ mutants define 41 vps complementation groups $^{\text {a }}$

\begin{tabular}{|c|c|c|}
\hline vps mutant & $v p t$ mutant & vpl mutant \\
\hline vps1 & vpt26 & vpl1 \\
\hline$v p s 2$ & vpt14 & vpl2 \\
\hline vps3 & vpt17 & vpl3 \\
\hline vps4 & vpt10 & vpl4 \\
\hline vps5 & vpt5 & - \\
\hline vps 6 & vpt 13 & vpl6 \\
\hline vps8 & vpt8 & vpl8 \\
\hline vps9 & vpt9 & vpl31 \\
\hline vps10 & vpt1 & - \\
\hline vps 11 & vpt11 & vpl9 \\
\hline vps 13 & vpt2 & - \\
\hline vps 15 & vpt15 & vpl19 \\
\hline vps16 & vpt16 & - \\
\hline vps17 & vpt3 & - \\
\hline vps18 & vpt18 & - \\
\hline vps19 & vpt19 & vpl21 \\
\hline vps 20 & vpt 20 & vpl10 \\
\hline vps 21 & vpt 21 & - \\
\hline vps 22 & vpt 22 & vpl14 \\
\hline vps 23 & vpt 23 & vpl15 \\
\hline vps 24 & vpt 24 & vpl26 \\
\hline vps 25 & vpt 25 & vpl12 \\
\hline vps 26 & vpt4 & - \\
\hline vps 27 & vpt 27 & vpl23 \\
\hline vps 28 & vpt 28 & vpl13 \\
\hline vps 29 & vpt6 & - \\
\hline vps30 & vpt 30 & - \\
\hline vps 31 & vpt31 & - \\
\hline vps32 & vpt32 & vpl5 \\
\hline vps33 & vpt33 & vpl 25 \\
\hline vps34 & vpt 29 & vpl7 \\
\hline vps35 & $v p t 7$ & - \\
\hline vps36 & - & vpl11 \\
\hline vps37 & - & vpl16 \\
\hline vps38 & - & vpl17 \\
\hline vps39 & - & vpl18 \\
\hline$v p s 41$ & - & vpl20 \\
\hline vps 43 & - & vpl24 \\
\hline$v p s 44$ & - & vpl27 \\
\hline$v p s 45$ & - & vpl 28 \\
\hline vps46 & - & vpl30 \\
\hline
\end{tabular}

a There have been several revisions to the list of vps mutants since the last compilation was published (Klionsky et al., 1990). They are as follows. 1) Ten new vpl complementation groups were identified that have not been previously published (vpl20 through vpl31, except $v p l 22$ and $v p l 29$, which were eliminated). Five of these $v p l$ groups overlap with established vps groups and five define five new $v p s$ complementation groups. 2) Four previously undetected overlaps between $v p l$ and vpt mutants were discovered. Based on comparison of restriction maps of cloned genes, VPS7 is the same as VPS34 and VPS40 is the same VPS15 (Herman and Emr, 1990; Herman et al., 1991; Raymond and Stevens, unpublished data). In addition, vps 2 mutants failed to complement vps14 mutants and vps12 mutants failed to complement vps 21 strains. When diploids from these crosses were sporulated and haploid progeny analyzed (from at least 15 four spore tetrads), the $\mathrm{Vps}^{-}$phenotype segregated 4:0. This demonstrates that both sets of overlaps are the consequence of allelic mutations in the same gene. 3) Diploids formed between several alleles of vpl5 and $v p t 5$ complemented one another, indicating that the two groups define separate genes. However, the vpl5 mutants failed to complement vpt32 mutants, and thus these two sets of mutants form one complementation group. To incorporate these changes vps $5=v p t 5$ and $v p s 32$ $=v p l 5=v p t 32$.

\section{Class A vps Mutants Have Vacuoles Similar to Those of Wild-type Cells}

The morphology of vacuoles in wild-type yeast strains and the changes the organelle undergoes during the growth of vegetative cells or on entry into stationary phase have been described extensively elsewhere (Weimken et al., 1970; Weisman et al., 1987; Weisman and Wickner, 1988; Pringle et al., 1989; Raymond et al., 1990; Gomes de Mesquita et al., 1991). The wild-type parent strains of the vps mutants (Table 1) all possess similar vacuolar morphologies (Figure 1, A-D; Raymond, 1990). The organelle generally appears to have 1-10 lobes that cluster together in one region of the cell. During the cell cycle, portions of the mother vacuole appear to actively partition into developing buds (Figure $1, A-D)$. The characteristic mother to bud vacuolar extensions have been termed vacuolar "segregation structures" (Raymond et al., 1990; Gomes de Mesquita et al., 1991), and the process whereby maternal vacuolar material is passed to daughter cells has been termed "vacuole inheritance" (Weisman et al., 1987, 1990; Weisman and Wickner, 1988; Shaw and Wickner, 1991). In wildtype cells, the ALP staining pattern and the $60-\mathrm{kDa}$ subunit staining pattern are virtually indistinguishable (Figure 1, A-D), indicative of the fact that both ALP and the V-ATPase reside predominantly on the vacuolar membrane.

Mutants from nine vps complementation groups were found to have vacuoles similar to wild-type, and in keeping with previous work these were designated as

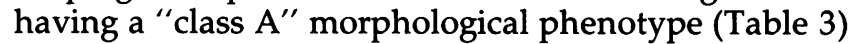
(Banta et al., 1988). In some mutants (vps10, vps13, vps44, vps46), there was no detectable difference between the vacuoles in the mutants and those in the wild-type parents. In the remaining mutant strains (vps8, vps 29 , vps 30 , vps 35, vps38), the morphology of the vacuole was slightly perturbed, as is shown for the vps 35 mutant in Figure 1, E-H. Most of these mutant cells appeared to have one to two vacuoles per cell with a single round vacuole per cell being the predominant morphology. Although vacuolar segregation structures were far less prominent in this set of mutants than in wild-type strains, all large buds possessed distinct vacuoles indicative of normal vacuole inheritance. Furthermore, antibodies directed against the $60-\mathrm{kDa}$ subunit of the V-ATPase decorated the vacuolar membrane, and quinacrine accumulation was normal in these class A vps mutants, suggesting that the V-ATPase complex was properly assembled. The amount of newly synthesized CPY secreted from the class A vps mutants was somewhat variable (Table 3 ), particularly among the strains with vacuoles that were most similar to wildtype.

\section{Class B vps Mutants Possess Fragmented Vacuoles}

Certain vps mutants displayed a fragmented vacuole phenotype. These strains were designated previously 

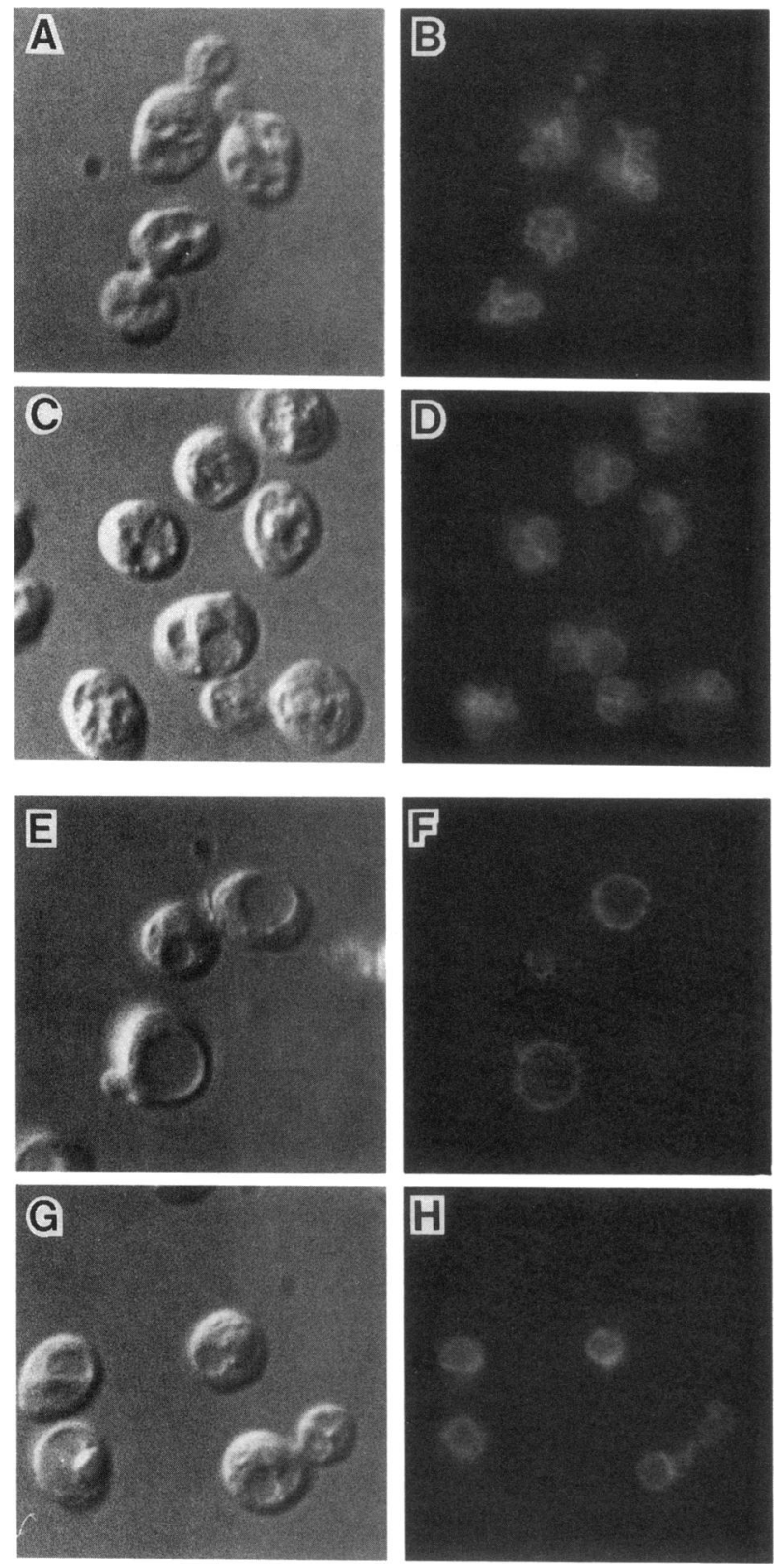

Figure 1. Vacuole morphology in the wild-type strain SF838-9D (A-D) and the class A mutant vps35 (vpt7; E-H). ALP staining is shown in $B$ and $F, 60-k D a$ V-ATPase subunit staining in D and $H$, and the corresponding Nomarski images of whole cells in A, C, E, and G. One centimeter equals $\sim 5 \mu \mathrm{m}$.

as class B vps mutants (Banta et al., 1988). Class B mutant cells had a large number of small vacuolar compartments that were visible by Nomarski optics and by staining with either ALP or $60-\mathrm{kDa}$ V-ATPase subunit antibodies (Figure 2). Quinacrine accumulated in multiple small compartments in all of the class B mutants, suggesting that functional V-ATPase was assembled onto the membranes of the fragmented vacuoles. How- ever, in certain class B mutants (vps41; Figure 2H), the diffuse appearance of the V-ATPase staining pattern may indicate a partial defect in the assembly of the VATPase. Mutants representing five complementation groups were designated class B (Table 3). One mutant complementation group originally designated as class B, vps1 (Banta et al., 1988), was similar to class B mutants but appeared to have a distinct phenotype as described in a later section.

Within the class B group of mutants, there appeared to be two subgroups. The many small vacuoles in $v p s 5$ and vps 17 mutants retained the wild-type characteristic of clustering within one region of the cell (Figure 2, AD). Inspection of a large number of labeled cells revealed that vacuole fragments in these mutants were often aligned along the mother cell to bud axis in a configuration reminiscent of wild-type vacuolar segregation structures. In contrast, the fragmented vacuoles in vps39, vps41, and vps 43 class B mutants appeared to be randomly dispersed throughout the cell (Figure 2, E-H). The class B vps mutants secreted a consistently high level of newly synthesized CPY (Table 3 ).

\section{Class C vps Mutants Lack Coherent Vacuoles}

Mutants from four vps complementation groups did not possess an organized vacuole (Table 3 ). These class $\mathrm{C}$ vps mutants (Banta et al., 1988) have been the subject of several investigations (Chvatchko et al., 1986; Banta et al., 1988, 1990; Dulic and Reizman, 1989; Wada et al., 1990; Woolford et al., 1990; Robinson et al., 1991; Preston et al., 1991). ALP staining of the class $C$ mutants yielded a scattered punctate pattern (Figure $3 B$ ), suggesting that this integral membrane protein may be distributed among the multiple vesicle-like structures seen in electron micrographs of these mutants (Chvatchko et al., 1986; Banta et al., 1988; Rothman et al., 1989a; Klionsky et al., 1990; Wada et al., 1990; Woolford et al., 1990). No cell surface staining with the ALP antibody was apparent, indicating that the majority of this vacuolar membrane protein remains sequestered intracellularly. The steady-state level of ALP in class C mutants was comparable with that seen in wild-type cells, as determined by quantitative Western blot analysis. Thus, the reduced fluorescence intensity of the ALP-specific signal in class $C$ mutants relative to other cells was probably a consequence of its highly dispersed intracellular distribution. The staining pattern of the $60-\mathrm{kDa}$ V-ATPase subunit was diffuse (Figure 3D). The subtle differences between certain class B vps mutants (Figure 2, E-H; vps39, vps41, and vps43) and the class $\mathrm{C}$ mutants (Figure 3) observed by indirect immunofluorescence using ALP and $60-\mathrm{kDa}$ V-ATPase subunit antibodies are more readily apparent using electron microscopic techniques (Banta et al., 1988). As reported previously, all four of the class $C$ vps mutants are strongly defective for CPY sorting (Table 3). 
Table 3. Morphological classification and CPY secretion among the vps mutants ${ }^{a}$

\begin{tabular}{|c|c|c|c|c|c|c|c|c|c|c|c|}
\hline Class A & $\%$ CPY & Class B & $\%$ CPY & Class C & $\%$ CPY & Class D & $\%$ CPY & Class E & $\%$ CPY & Class F & $\%$ CPY \\
\hline $\begin{array}{l}\text { vps8 } \\
\text { vps10 } \\
\text { vps } 13 \\
\text { vps } 29 \\
\text { vps30 } \\
\text { vps35 } \\
\text { vps38 } \\
\text { vps44 } \\
\text { vps } 46\end{array}$ & $\begin{array}{l}66,67 \\
62,84 \\
21,30 \\
73,68 \\
66,72 \\
67,81 \\
62,59 \\
40,34 \\
27,23\end{array}$ & $\begin{array}{l}\text { vps5 } \\
\text { vps17 } \\
\text { vps39 } \\
\text { vps41 } \\
\text { vps43 }\end{array}$ & $\begin{array}{l}97,69 \\
74,79 \\
57,44 \\
58,31 \\
52,34\end{array}$ & $\begin{array}{l}\text { vps11 } \\
\text { vps16 } \\
\text { vps18 } \\
\text { vps33 }\end{array}$ & $\begin{array}{c}84,80 \\
69 \\
79 \\
72,86\end{array}$ & $\begin{array}{l}\text { vps3 } \\
\text { vps6 } \\
\text { vps9 } \\
\text { vps15 } \\
\text { vps19 } \\
\text { vps21 } \\
\text { vps34 } \\
\text { vps45 }\end{array}$ & $\begin{array}{l}69,65 \\
67,70 \\
58,45 \\
67,72 \\
77,68 \\
35,23 \\
54,53 \\
71,69\end{array}$ & $\begin{array}{l}\text { vps2 } \\
\text { vps4 } \\
\text { vps20 } \\
\text { vps22 } \\
\text { vps23 } \\
\text { vps24 } \\
\text { vps25 } \\
\text { vps27 } \\
\text { vps28 } \\
\text { vps31 } \\
\text { vps32 } \\
\text { vps36 } \\
\text { vps37 }\end{array}$ & $\begin{array}{r}30,30 \\
43,48 \\
48,45 \\
21,29 \\
31,31 \\
29,40 \\
34,30 \\
33,43 \\
37,38 \\
9,16 \\
33,54 \\
39,37 \\
28,20\end{array}$ & $\begin{array}{l}\text { vps1 } \\
\text { vps26 }\end{array}$ & $\begin{array}{l}78,82 \\
72,76\end{array}$ \\
\hline
\end{tabular}

a \% CPY represents the percentage of newly synthesized CPY that is secreted from each ${ }^{35} \mathrm{~S}$ labeled vps mutant, as determined by quantitative immunoprecipitations and determination of the amount of radioactive CPY in the intracellular and extracellular fractions. The pairs of numbers are the percentage of newly synthesized material secreted from the MAT $\alpha$ and $M A T a$ alleles of each $v p s$ mutant used in this study, respectively.

\section{Class D vps Mutants Display Defects in Vacuole Inheritance and Acidification of the Vacuole}

The class $\mathrm{D}$ vps mutants have been found to exhibit defects in several aspects of vacuole assembly. Two members of this group (vps3 and vps6) initially attracted interest because they were unable to generate a $\mathrm{pH}$ gradient across the vacuolar membrane, and they failed to assemble peripheral membrane subunits of the $\mathrm{V}$ ATPase onto the cytoplasmic surface of the vacuole (Rothman et al., 1989b). The latter phenotype is illustrated for vps 45 in Figure 4D, where antibodies for the $60-\mathrm{kDa}$ V-ATPase subunit, which is present at wildtype levels, produced a diffuse cytoplasmic staining pattern. Further characterization of vps 3 and vps $34 \mathrm{mu}-$ tants revealed that these mutants are also defective for vacuole inheritance (Herman and Emr, 1990; Raymond et al., 1990). The vacuoles in the mother cells of class D vps mutants appeared as single large spheres that failed to form segregation structures (Figure 4, B and F). Buds appeared to receive little or no vacuolar material from mother cells, and antibodies against ALP sometimes produced dispersed punctate staining patterns in daughter cells, similar to class $C$ cells (Raymond et al., 1990). This suggests that nascent vacuolar material produced in these cells transiently accumulates as unfused vesicles. The fact that nearly all mother cells possess vacuoles raises the possibility that vacuoles can arise de novo from unassembled vacuole precursors within daughter cells.

Mutants from eight $v p s$ complementation groups displayed class $D$ phenotypes. Independent screens produced four additional vacuole segregation (vac) mutants with defects in vacuole inheritance (Weisman et al., 1990; Shaw and Wickner, 1991; Weisman and Wickner, 1992). However, complementation testing between vac and vps mutants identified vac1 as vps19, vac3 as vps 36 , and vac4 as vps 15 .

Within the class D vps strains there appeared to be subgroups of mutants with somewhat distinct characteristics. Yeast strains carrying vps3, vps6, vps9, vps21, or vps 45 mutations had phenotypes indistinguishable from those of vps $3 \Delta$ strains described previously (Figure 4, A-D) (Raymond et al., 1990). The morphological phenotypes of vps 15 and vps 34 mutants (Figure 4, E$\mathrm{H})$, although indistinguishable from one another, were slightly different from other class $D$ mutants. In addition to vacuolar membrane staining, antibodies against ALP yielded punctate cytoplasmic labeling in almost all vps15 and vps 34 cells, and the percentage of (daughter) cells that lacked apparent vacuoles and had a class $C$-like staining pattern was several-fold higher than in other class D mutants (Figure 4F). These data are consistent with the interpretation that ALP is present in the membranes of the multiple vesicular bodies seen in electron micrographs of these mutants (Banta et al., 1988). Furthermore, antibodies that recognize the $60-\mathrm{kDa} \mathrm{V}$ ATPase subunit labeled the vacuolar membrane and the cytoplasm in vps 15 and vps 34 strains, indicating that the V-ATPase assembly defect in these mutants was less pronounced than in other class D strains (compare Figure 4, D and $\mathrm{H}$ ). In support of this, measurements of V-ATPase specific activities in isolated vacuolar membranes prepared from vps 34 strains were $20-40 \%$ of the wild-type levels, significantly higher than the levels measured in vps3 and vps6 mutants (Rothman et al., 1989b; Yamashiro, 1990). The similarity between vps15 and vps34 mutants is consistent with the finding that Vps15p and Vps34p interact physically and that genetic interaction between these genes has been observed (Herman et al., 1991). All of the class D vps mutants secreted high percentages of newly synthesized 

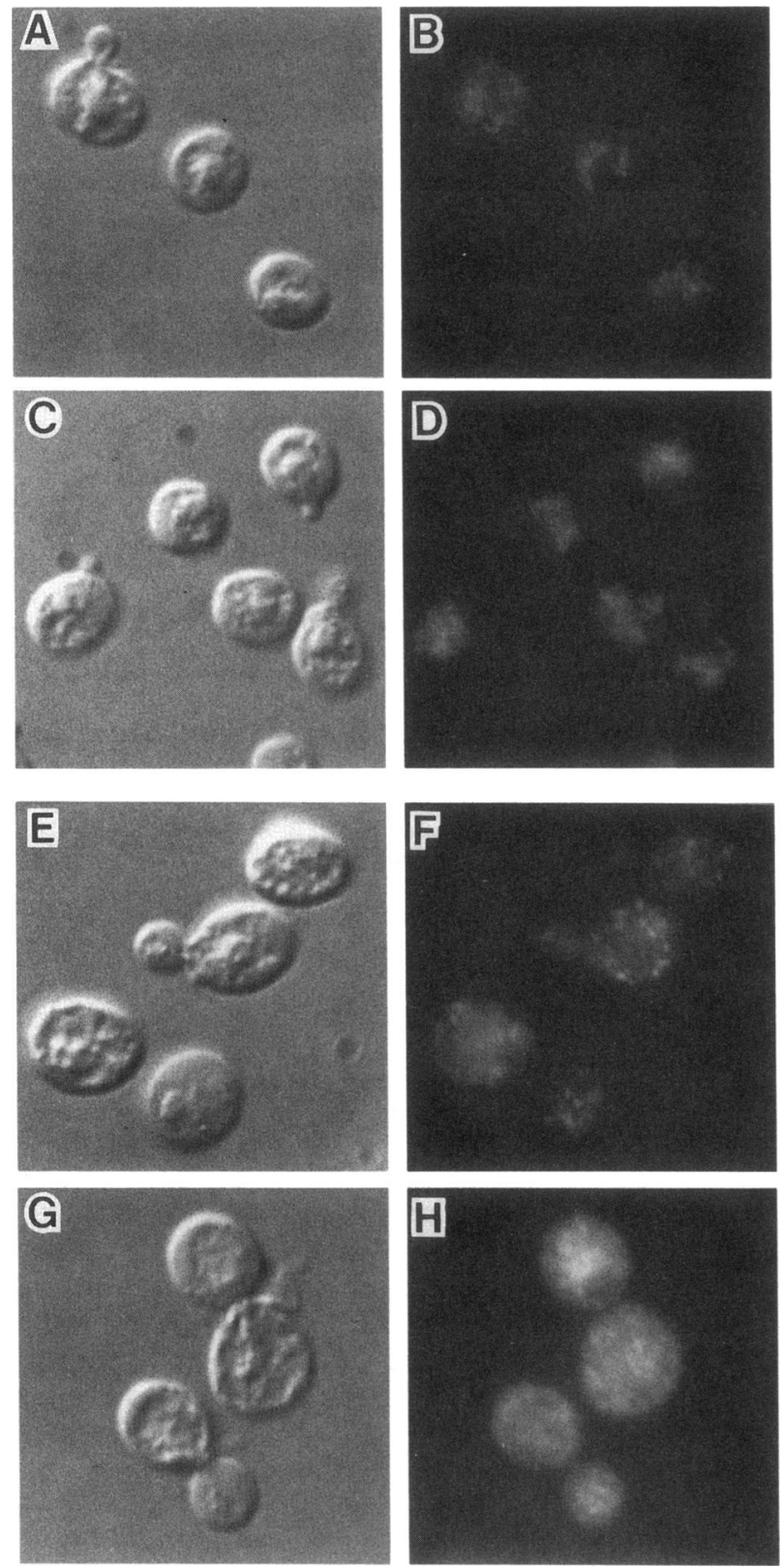

Figure 2. Class B mutants vps17 (vpt3; A-D) and vps41 (vpl20-6; E$\mathrm{H})$ exhibit similar but distinct classes of fragmented vacuoles. ALP staining is shown in $\mathrm{B}$ and $\mathrm{F}, 60-\mathrm{kDa}$ vacuolar $\mathrm{H}^{+}$-ATPase subunit staining in $\mathrm{D}$ and $\mathrm{H}$, and the corresponding Nomarski images of whole cells in A, C, E, and G. One centimeter equals $\sim 5 \mu \mathrm{m}$.

CPY except for vps21 mutants (Table 3). The morphological phenotypes of vps 21 strains are otherwise identical with the subgroup of mutants that includes vps3.

\section{A Novel Prevacuolar-like Organelle is Prominent in Class E vps Mutants}

Antibodies directed against ALP, as well as visualization of vacuoles by Nomarski optics, revealed that the vac- uoles of class $\mathrm{E}$ vps mutants were relatively normal in appearance (Figure 5, A and B). Antibodies specific for the $60-\mathrm{kDa}$ V-ATPase subunit revealed some vacuolar and diffuse staining, but the majority of protein appeared to be sequestered in a separate compartment (termed the "class E compartment") that was distinct from the vacuole (Figure 5, C and D). Two lines of evidence suggested that the extravacuolar $60-\mathrm{kDa} \mathrm{V}$ ATPase subunit was part of active V-ATPase complexes that were clustered around the class E compartment. First, quinacrine accumulated in the vacuole at low levels, but very high levels accumulated in the smaller class E compartment(s) adjacent to the vacuole (Figure 6, A and B). Quinacrine labeling of the extravacuolar compartment in class E vps mutants was sensitive to the addition of $200 \mathrm{mM}$ ammonium acetate (Raymond, 1990), suggesting that quinacrine accumulated in the extravacuolar compartment because it was acidified (Weisman et al., 1987; Rothman et al., 1989b). Second, the V-ATPase specific activities measured in membranes prepared from several class E mutants were very similar to the specific activity measured in wild-type membranes (Yamashiro, 1990; Yamashiro, Rothman, and Stevens, unpublished observation).

The distinct distributions of ALP and the $60-\mathrm{kDa} \mathrm{V}$ ATPase subunit in class $E$ cells suggested that the novel class E compartment defined by the V-ATPase may represent exaggerated prevacuolar or endosome-like organelles. This hypothesis was investigated further by examining the distribution of a membrane protein of
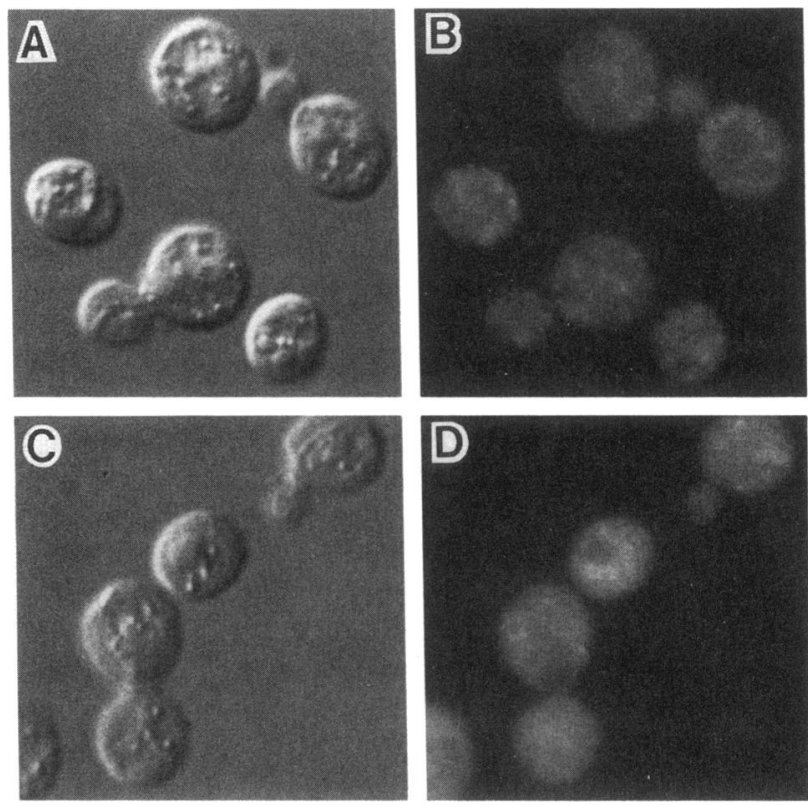

Figure 3. The class $C$ mutant vps11 (vpl19-13) lacks organized vacuolar structures. ALP staining is shown in B, $60-\mathrm{kDa}$ V-ATPase subunit staining in $D$, and the corresponding Nomarski images of whole cells in $\mathrm{A}$ and $\mathrm{C}$. One centimeter equals $\sim 5 \mu \mathrm{m}$. 

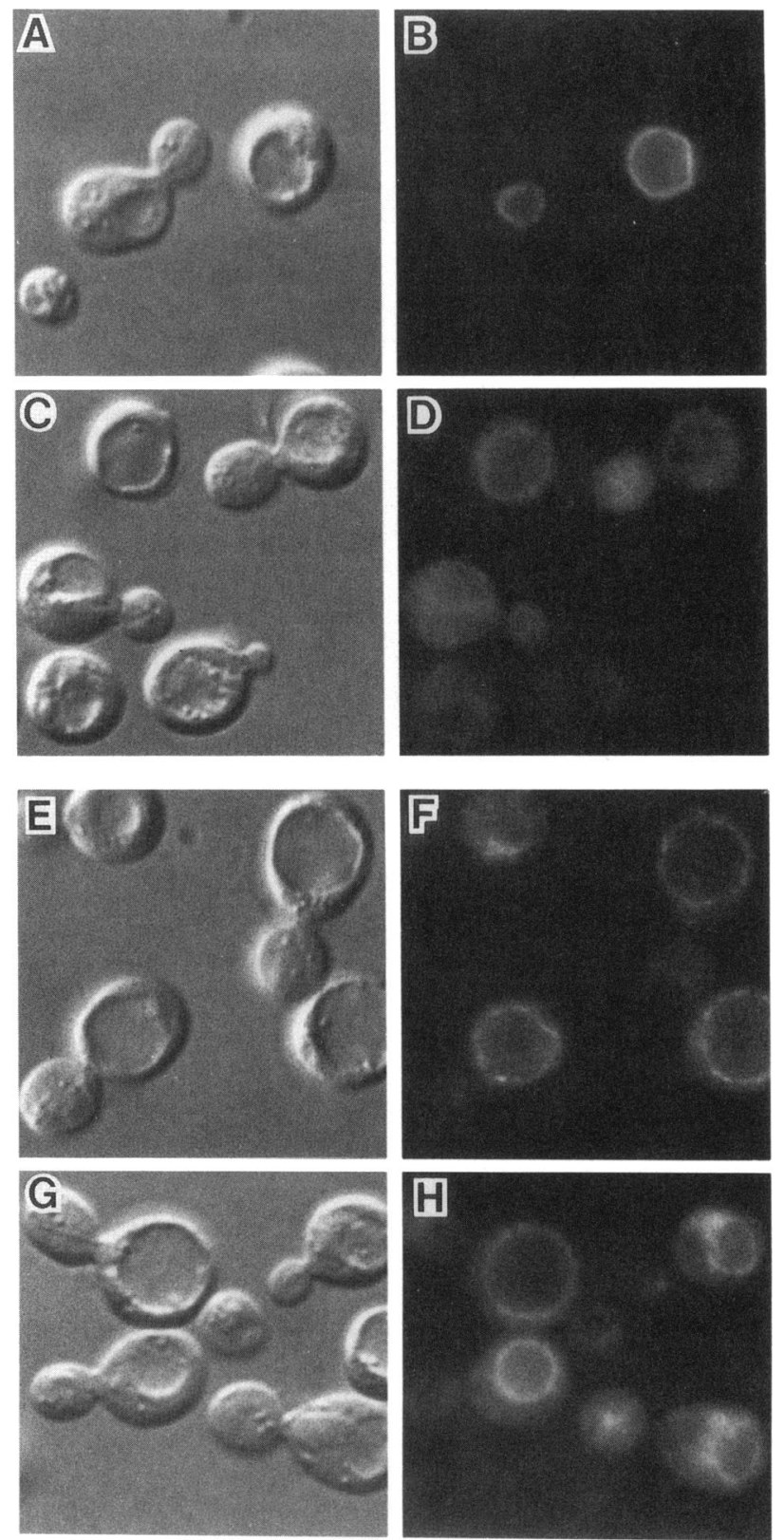

Figure 4. Class D mutants vps45 (vpl28-4; A-D) and vps15 (vpl19$10 ; \mathrm{E}-\mathrm{H}$ ) exhibit defects in vacuole inheritance and assembly of the 60-kDa subunit of the V-ATPase onto the vacuolar membrane. ALP staining is shown in B and F, 60-kDa V-ATPase subunit staining in $\mathrm{D}$ and $\mathrm{H}$, and the corresponding Nomarski images of whole cells in $\mathrm{A}, \mathrm{C}, \mathrm{E}$, and $\mathrm{G}$. One centimeter equals $\sim 5 \mu \mathrm{m}$.

the late-Golgi compartment and soluble vacuolar proteins in class $E$ mutant cells using indirect immunofluorescence (Figure 7). The late-Golgi marker was an integral membrane fusion protein that carries the cytoplasmic tail of dipeptidyl aminopeptidase A (DPAP A) fused to the transmembrane and lumenal domains of ALP (A-ALP). This protein was shown to reside in
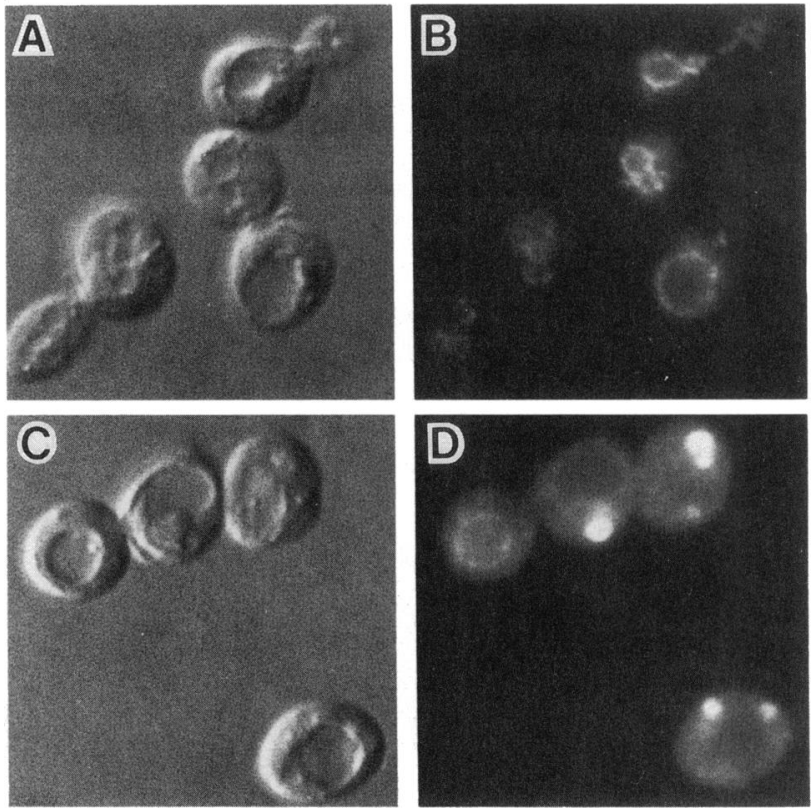

Figure 5. The class E mutant vps27 (vpl23-5) possesses a novel 60$\mathrm{kDa}$ V-ATPase subunit stained organelle distinct from the ALP staining vacuole. ALP staining is shown in B, $60-\mathrm{kDa} \mathrm{V}$-ATPase subunit staining in $\mathrm{D}$, and the corresponding Nomarski images of whole cells in $\mathrm{A}$ and $\mathrm{C}$. One centimeter equals $\sim 5 \mu \mathrm{m}$.

the late-Golgi (as defined by Kex $2 \mathrm{p}$ co-localization) (Redding et al., 1991) compartment of wild-type cells (Roberts, 1991; Roberts et al., 1992; Nothwehr and Stevens, unpublished observations). In class E mutants, a significant portion of the A-ALP fusion protein was localized to the novel class E compartment, as demonstrated in the double-label immunofluorescence images shown in Figure 7, A-C. The remaining signal from the A-ALP fusion protein appears localized to punctate regions typical of Golgi staining in wild-type yeast cells (Redding et al., 1991). Similar double-labeling procedures with antibodies that recognize CPY and antibodies
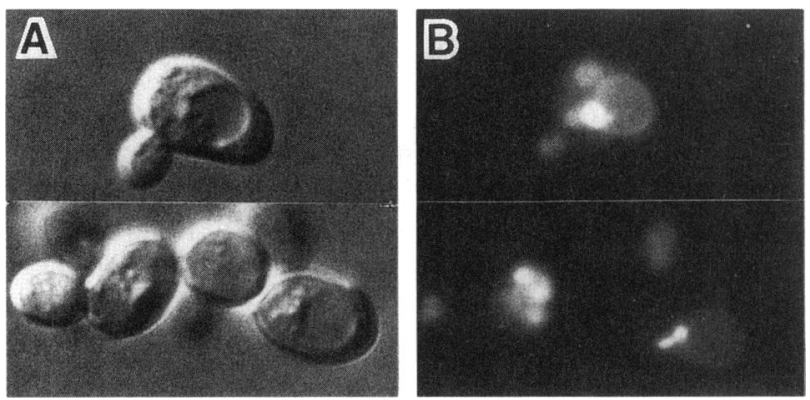

Figure 6. Quinacrine accumulates to high levels in a novel compartment of the class E mutant vps27 (vpl23-5). Quinacrine staining is shown in B and the corresponding Nomarski image of whole cells in $\mathrm{A}$. One centimeter equals $\sim 5 \mu \mathrm{m}$. 

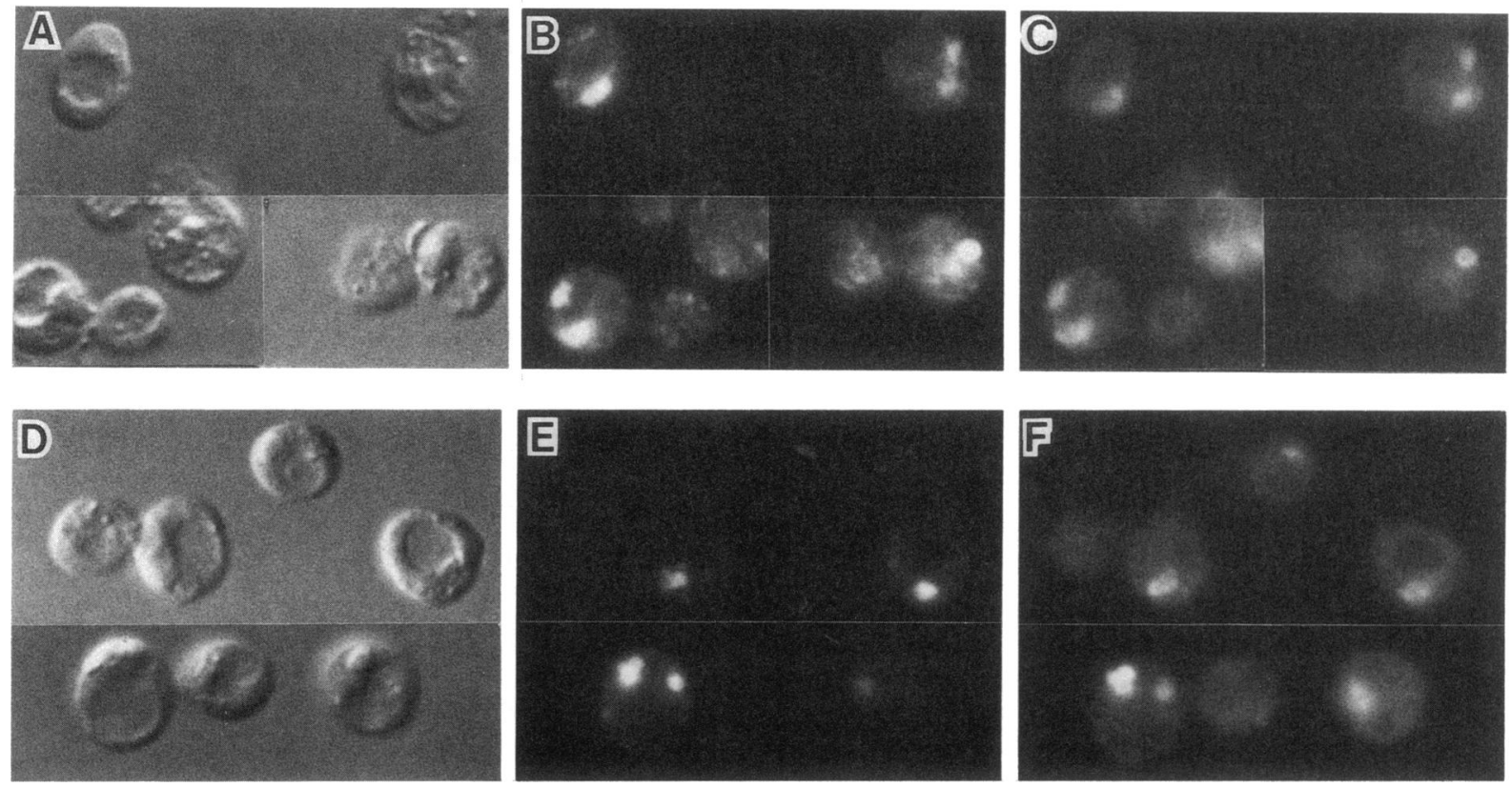

Figure 7. Double-labeling experiments demonstrate that significant pools of A-ALP and CPY, proteins normally found in the late Golgi and the vacuoles of wild-type cells, respectively, accumulate in the novel prevacuolar organelle of the class E mutant vps27 (CRY2-8A, A-C; vpl23$5, \mathrm{D}-\mathrm{F})$. The distribution of the A-ALP fusion construct is revealed by ALP antibodies in B. Note that the yeast strain shown is a vps27 $\Delta p h o 8 \Delta$ double mutant (Table 1), and thus the endogenous vacuolar signal from ALP is absent. CPY staining is shown in E. Both proteins colocalize with the 60-kDa V-ATPase subunit, which defines the novel class E compartment, as shown in C and F. Nomarski images are shown in A and D. One centimeter equals $\sim 5 \mu \mathrm{m}$.

for the 60-kDa V-ATPase subunit showed that most of the intracellular CPY also is localized within the class E compartment (Figure 7, D-F). The soluble vacuolar proteins proteinase $A(\operatorname{PrA})$ and proteinase $B$ were also found in the novel class $E$ organelle. The observations presented in Figure 7 indicate that the novel class E compartment possesses marker proteins of both the lateGolgi and the vacuole, consistent with the interpretation that it is an exaggerated prevacuolar organelle.

Mutants from thirteen vps complementation groups exhibited a class E phenotype (Table 3). All of the class E vps mutants secreted modest amounts of newly synthesized CPY (Table 3).

\section{Vacuoles in Class F vps Mutants are Encircled by Smaller Vacuolar Compartments}

Mutants from two vps complementation groups, vps1 and vps 26, have vacuolar morphologies intermediate between wild-type cells and the vacuolar morphology of class B mutants (Figure 8); vps1 mutants were, in fact, originally described as class B mutant strains (Banta et al., 1988). Unlike class B strains, however, cells in both class $\mathrm{F}$ mutants typically contain a large central vacuole (Figure 8, A and C). The labeling pattern of ALP and 60-kDa V-ATPase subunit reveal that this central compartment is often surrounded by smaller vacuole-like compartments (Figure 8, B and D). Although segregation structures are seldom observed in vps 1 and vps 26 cells, large buds contain vacuoles indicating that vacuole inheritance occurs in these strains. The observations that the $60-\mathrm{kDa}$ subunit is assembled onto membranes and that vacuoles from both mutants label with quinacrine (Banta et al., 1988; Rothman et al., 1989b) suggest that V-ATPase assembly is not influenced by the vps1 or the vps 26 mutations. Both class F mutants exhibited severe CPY sorting defects (Table 3).

\section{DISCUSSION}

Indirect immunofluorescence microscopy was used to characterize the vacuolar morphology in vps mutants representing 41 complementation groups. As with previous studies (Banta et al., 1988; Rothman et al., 1989a), we encountered $v p s$ mutants that exhibited profound perturbations in vacuole assembly, and we found that groups of mutants from different complementation groups shared similar aberrant morphological phenotypes. However, the major conclusion drawn in the previous reports was that majority of vps mutants had wild-type class $\mathrm{A}$ vacuoles. The greater resolution of the analytical tools utilized in this study indicates that many of the mutants originally placed in the class $\mathrm{A}$ group have vacuoles that are significantly different from the wild-type organelle. Once again, groups of mutants from different complementation groups were found to 

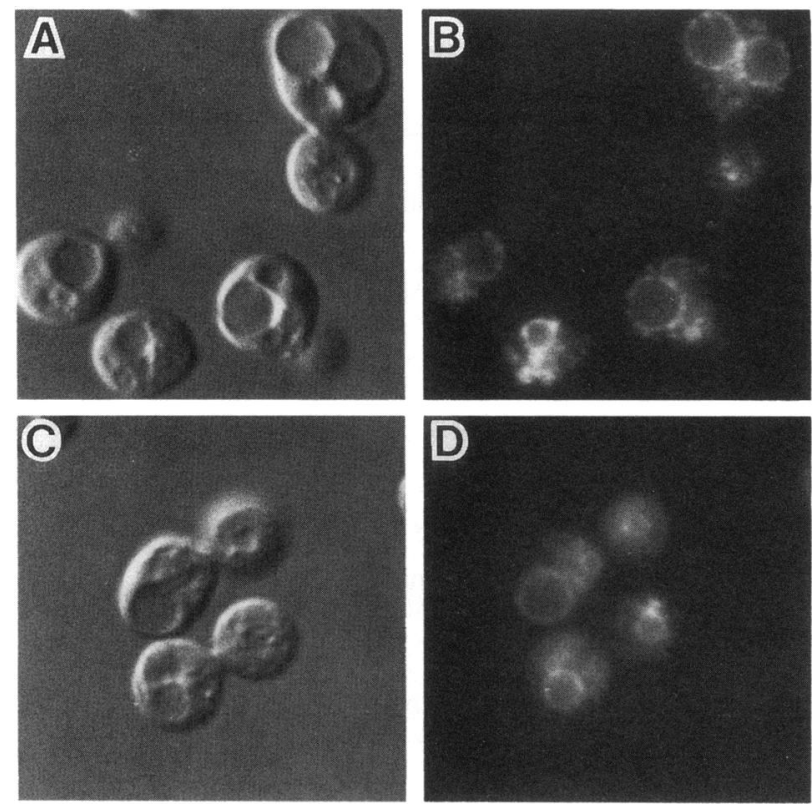

Figure 8. Vacuole-like compartments cluster around a central vacuoles in the class F mutant vps1 (SF838-9D vpsl- $\Delta 2$ ). ALP staining is shown in B, 60-kDa V-ATPase subunit staining in D, and the corresponding Nomarski images of whole cells in A and C. One centimeter equals $\sim 5 \mu \mathrm{m}$.

share similar traits, and so the class A, class B, and so on, nomenclature of Banta et al. (1988) was extended to include these new groups. Overall, six classes of distinct morphological phenotype could be differentiated among mutants of the 41 vps complementation groups (Table 4). Quantitative analysis of the CPY missorting defects in the same collection of mutants suggests there was, in most cases, a good correlation between morphological phenotype and the severity of the sorting defect within a given class of vps mutants.

Our findings represent a significant contribution toward understanding vacuolar biogenesis in S. cerevisiae and thus to the more general problem of organelle assembly in eukaryotic cells. The analysis presented here demonstrates that most vps mutants can be differentiated from their isogenic wild-type parents based on morphological phenotypes and that groups of mutants appear to share similar aberrant characteristics. Many of the phenotypic features of individual class A, B, C, $\mathrm{D}$, and $\mathrm{F}$ vps mutants have been described previously (reviewed in Klionsky et al., 1990; Raymond et al., 1992a), but an overview of the entire vps mutant collection that accounted for newly discovered morphological phenotypes (e.g., class D, E, and F phenotypes) had not been conducted. An important conclusion drawn from the current analysis is that this large collection of vps mutants can be subdivided into a relatively small number of morphological groups. The fact that there are at least six distinct classes of vps mutants sug- gests there also may be at least six "pathways" or distinct biochemical "processes" required for vacuolar protein sorting and vacuole assembly. As a corollary, the wildtype gene products affected in mutants that share similar phenotypes may act in the same pathway. This is exemplified by the vps 15 and vps 34 mutants. These mutants share identical vacuolar morphologies, and the wild-type Vps15p and Vps34p products appear to act as a complex (Herman et al., 1991).

The other significant aspect of this work is that the morphological characteristics of a given mutant suggest possible functions for corresponding wild-type gene products. For example, mutations that specifically affect the soluble protein sorting apparatus would not be anticipated to perturb other events in vacuole assembly, and, thus, such mutants might be expected to have a class A phenotype. Mutations in a putative soluble vacuolar protein sorting receptor complex eventually may be identified among the class A vps mutants, particularly those with a pronounced sorting defect.

In class $B$ mutants there are many small vacuolar compartments, and in class $C$ mutants there are no apparent vacuoles, yet no cell surface ALP staining was observed in these classes (or any other class) of vps mutants. These data underscore the fundamental differences between the sorting of soluble and integral membrane vacuolar proteins. Recent evidence suggests that membrane proteins lacking specific sorting information are delivered to the vacuole by default (Roberts, 1991; Cooper and Bussey, 1992; Raymond et al., 1992a,b; Roberts et al., 1992; Wilcox et al., 1992). The fact that none of the vps mutations results in delivery of ALP to the cell surface, even in class $C$ mutants, which have an extreme vacuole assembly phenotype, supports this conclusion.

The phenotype of class E vps mutants, which accumulate a novel compartment with protein constituents from both the late Golgi and the vacuole, has not been described previously. These mutants secrete modest amounts of newly synthesized CPY in its precursor form, and the CPY that is retained intracellularly is processed to its mature form (see Rothman and Stevens, 1986; Robinson et al., 1988; Vater, Raymond, and Stevens, unpublished observations). Because a large pool of CPY was found within the novel class E compartment, by inference this material is in its processed enzymatically active form. This supposition is supported by the fact that the A-ALP fusion protein, which contains a C-terminal propeptide segment that is proteolytically cleaved by vacuolar hydrolases (Klionsky and Emr, 1989), partially localizes to the class E compartment and is partially processed in the class E mutant vps27 (Raymond, Nothwehr, and Stevens, unpublished data). These data argue that the class $E$ compartment is a post-Golgi organelle and not an exaggerated form of the late Golgi because only $\mathrm{p} 2$ precursor form of CPY 
Table 4. Phenotypic characteristics of the morphological groups that define the vps mutants

\begin{tabular}{|c|c|}
\hline Class & Characteristics \\
\hline Class A & $\begin{array}{l}\text { Wild-type vacuolar morphology. Vacuoles frequently appear as } 3-10 \text { subcompartments that cluster to one region of the } \\
\text { cytoplasm. In premitotic budding cells, the vacuole is likely to extend between mother cell and developing bud along the cell } \\
\text { axis. ALP and V-ATPase staining patterns of the vacuole are superimposable. }\end{array}$ \\
\hline Class B & $\begin{array}{l}\text { Mutants have highly "fragmented" vacuoles exhibiting }>20 \text { vacuole-like compartments per cell. In some mutants, these } \\
\text { subcompartments are clustered, whereas in others they are distributed randomly throughout the cell. ALP and V-ATPase } \\
\text { staining does not appear particularly discrete, owing in part to the dispersed nature of the vacuole-like compartments. } \\
\text { Quinacrine staining in the class B vps mutants sugests that some active V-ATPase complexes are assembled. }\end{array}$ \\
\hline Class C & $\begin{array}{l}\text { Mutant cells do not have an organelle readily identifiable as a vacuole. Multiple impressions visible by Nomarski optics are } \\
\text { presumably a mixture of vesicle-like organelles and lipid droplets seen previously in electron microscopy studies (Banta et al., } \\
\text { 1988; Wada et al., 1990). ALP staining is distinctly punctate, suggesting that this membrane protein is distributed among } \\
\text { vesicle-like membrane compartments. V-ATPase staining is more diffuse, suggesting that class C mutants are at least partially } \\
\text { defective for the assembly of the V-ATPase. }\end{array}$ \\
\hline Class D & $\begin{array}{l}\text { These mutants are defective for vacuolar segregation, meaning mother cells have a large, single, spherical vacuolar compartment } \\
\text { that fails to extend into developing buds, and newly formed daughter cells often lack coherent vacuoles and assume the } \\
\text { appearance of class C mutants (Raymond et al., 1990; Weisman et al., 1990; Shaw and Wickner, 1991). The V-ATPase fails to } \\
\text { assemble properly onto the vacuolar membrane, resulting in diffuse cytoplasmic localization of peripheral membrane V- } \\
\text { ATPase subunits. }\end{array}$ \\
\hline Class E & $\begin{array}{l}\text { ALP antibodies label vacuoles that are readily visible by Nomarski optics, whereas V-ATPase antibodies label a prominent } \\
\text { organelle distinct for the vacuole and from ALP staining structures; V-ATPase labeling is also weakly evident around the } \\
\text { ALP-staining vacuole and as diffuse staining throughout the cytoplasm. Significant portions of soluble vacuolar hydrolases } \\
\text { (CPY and PrA) and Golgi membrane proteins (DPAP A and A-ALP) also localize to the novel organelle in class E mutants. } \\
\text { These data indicate that class E mutants may possess an exaggerated form of a prevacuolar endosome-like compartment. }\end{array}$ \\
\hline Class F & $\begin{array}{l}\text { These mutants often have a large central vacuole surrounded by a number of class B-like fragmented vacuolar structures. As } \\
\text { such, these mutants exhibit vacuolar morphology distinct from class A and class B cells. ALP and V-ATPase antibodies yield } \\
\text { superimposable staining patterns. }\end{array}$ \\
\hline
\end{tabular}

is secreted, yet mature CPY is present in this novel compartment.

Class E vps mutants possess two distinct organelles with vacuolar markers, and the question arises as to which organelle is the vacuole and how these separate compartments are generated. The large organelle that is apparent by Nomarski optics and that also stains with ALP antibodies has the appearance normally associated with a yeast vacuole. On the other hand, the new compartment has active V-ATPase complexes and large pools of active vacuolar hydrolases associated with it. We envision at least two models for how these compartments are generated (Figure 9). In the first model, proteins sorted to a common vacuolar compartment in wild-type cells enter one of (at least) two separate sorting pathways (Figure 9A). These pathways converge in the vacuole in wild-type cells. In class $E$ mutants, these pathways fail to merge and lead to the generation of separate compartments. To accomodate experimental observations, it is also necessary to postulate that late Golgi proteins (e.g., A-ALP) enter one of these divergent pathways as a consequence of the class $\mathrm{E}$ vps mutation.

In the second model, proteins sorted in the late Golgi and bound for the vacuole enter a prevacuolar or endosome-like compartment. Furthermore, proteins that function in the late Golgi are hypothesized to enter the prevacuolar compartment, and in wild-type cells, these proteins are recycled back to the Golgi. Vacuolar proteins, on the other hand, are transported from the class
E compartment to the vacuole. In class $\mathrm{E}$ vps mutants, protein traffic from the prevacuolar endosome-like organelle is restricted for most of these proteins. This leads to the accumulation of proteins in the prevacuolar compartment and the morphological exaggeration of the organelle itself. We cannot, at present, distinguish between these models. However, evidence has been presented that wild-type cells possess a distinct compartment along the endocytic pathway (Singer and Riezman, 1990).

Two additional observations lead us to favor the interpretation that the class E compartment is a prevacuolar endosome-like organelle. Antibodies directed against Vps1p, which is thought to associate with the yeast Golgi but does not appear to associate with vacuolar membranes (Raymond, 1990; Rothman et al., 1990), label the class E organelle (Raymond and Stevens, unpublished observations). In addition, the a-factor receptor, Ste3p, which is endocytosed from the cell surface and delivered to the vacuole in wild-type strains, accumulates in the prevacuolar endosome-like organelle of the class E mutant vps2 (Davis and Sprague, personal communication). The fact that there are 13 class $\mathrm{E} v p s$ complementation groups indicates that a large number of components are required for conversion of the class $E$ organelle into normal wild-type vacuoles.

In summary, our work has shown that the large collection of vps mutants is composed of subclasses of mutant strains that share similar morphological phenotypes and CPY sorting defects. These data support a model 
A Wild-type

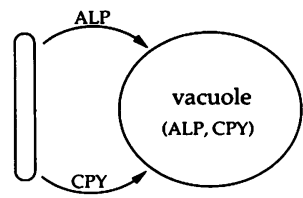

late Golgi

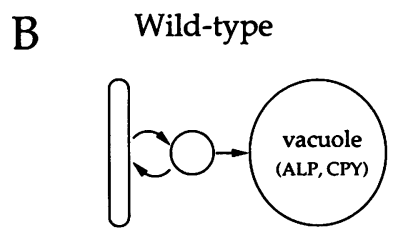

late Golgi
Class E vps

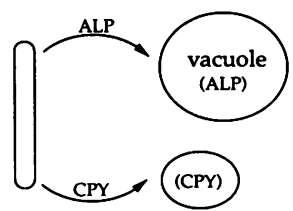

late Golgi Class E compartment

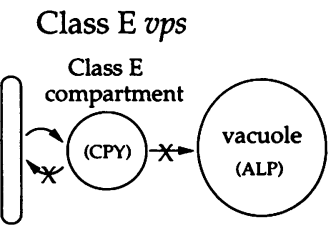

late Golgi

Figure 9. Two models for the formation of the new compartment in class E vps mutants. (A) Proteins bound for the vacuole in wildtype cells are normally packaged into at least two separate classes of transport vesicles that subsequently fuse with the vacuole. In class $E$ mutants, these pathways fail to merge. In addition, late Golgi proteins such as A-ALP enter the pathway defined by CPY as a consequence of the class E vps mutation. (B) Proteins bound for the vacuole in wild-type cells normally pass through a prevacuolar endosome-like compartment. Late Golgi proteins are hypothesized to cycle between the late Golgi compartment and the prevacuole. In class $\mathrm{E}$ mutants, most protein traffic (an exception being ALP) from the putative endosome-like organelle either to the vacuole or back to the late Golgi is restricted, leading to morphological exaggeration of the organelle. The steady-state resident proteins for each compartment are shown in parenthesis.

in which the different classes of mutants affect specific and discrete processes, pathways, or steps required for vacuole biogenesis. Similarly, mutants with indistinguishable morphological phenotypes are likely to act along the same or similar vacuole assembly pathway. These observations should facilitate the choices of VPS genes that are subjected to further molecular characterization. Finally, we have discovered that a large number of class $\mathrm{E}$ vps mutants possess a novel organelle that contains proteins from the late Golgi, the vacuole and a protein endocytosed from the cell surface. These are the characteristics expected of a prevacuolar endosomal compartment, and they support previous evidence that wild-type yeast cells possess endosomes (Singer and Riezman, 1990).

\section{ACKNOWLEDGMENTS}

We thank Cynthia Bauerle, Chris Roberts, Steve Nothwehr, and Nick Davis for stimulating discussion and encouragement; Jerry Gleason and Susan Detroy for production of photographs; and members of the Stevens' lab for commentary.

This work was supported by a National Institutes of Health predoctoral traineeship to C.K.R., by a Damon Runyon-Walter Winchell Cancer Research Fund award (DRG1012) to C.A.V., by a grant from the National Institutes of Health (GM-32448) to T.H.S., and by an American Cancer Society Faculty Research Award to T.H.S.

\section{REFERENCES}

Bankaitis, V.A., Johnson, L.M., and Emr, S.D. (1986). Isolation of yeast mutants defective in protein sorting to the vacuole. Proc. Natl. Acad. Sci. USA 83, 9075-9079.

Banta, L.M., Robinson, J.S., Klionsky, D.J., and Emr, S.D. (1988). Organelle assembly in yeast: characterization of yeast mutants defective in vacuolar biogenesis and protein sorting. J. Cell Biol. 107, 13691383.

Banta, L.M., Vida, T.A., Herman, P.K., and Emr, S.D. (1990). Characterization of yeast $\mathrm{Vps} 33 \mathrm{p}$, a protein required for vacuolar protein sorting and vacuole biogenesis. Mol. Cell. Biol. 10, 4638-4649.

Casadaban, M.F., and Cohen, S.N. (1980). Analysis of gene control signals by DNA fusion and cloning in Escherichia coli. J. Mol. Biol. 138, 179-207.

Chvatchko, Y., Howald, I., and Riezman, H. (1986). Two yeast mutants defective in endocytosis are defective in pheromone response. Cell 46, 355-364.

Cooper, A., and Bussey, H. (1992). Yeast Kex1p is a Golgi-associated membrane protein: deletions in a cytoplasmic targeting-domain result in mislocalization to the vacuolar membrane. J. Cell Biol. 119 (in press).

Dulic, V., and Riezman, H. (1989). Characterization of the END1 gene required for vacuole biogenesis and gluconeogenic growth of budding yeast. EMBO J. 8, 1349-1359.

Gomes de Mesquita, D.S., ten Hoopen, R., and Woldringh, C.L. (1991) Vacuolar segregation to the bud of Saccharomyces cerevisiae: an analysis of morphology and timing in the cell cycle. J. Gen. Microbiol. 137, 2447-2454.

Graham, T.R., and Emr, S.D. (1991). Compartmental organization of Golgi specific protein modification and vacuolar protein sorting events defined in a yeast sec18 (NSF) mutant. J. Cell Biol. 114, 207-218.

Herman, P.K., and Emr, S.D. (1990). Characterization of VPS34, a gene required for vacuolar protein sorting and vacuole segregation in Saccharomyces cerevisiae. Mol. Cell. Biol. 10, 6742-6754.

Herman, P.K., Stack, J.H., DeModena, J.A., and Emr, S.D. (1991). A novel protein kinase homolog essential for protein sorting to the yeast lysosome-like vacuole. Cell 64, 425-437.

Hirsch, J.J., Schiffer, H.H., Muller, H., and Wolf, D.H. (1991). Biogenesis of the yeast vacuole (lysosome): mutation in the active site of the vacuolar serine protease $\mathrm{yscB}$ abolishes proteolytic maturation of its 73-kDa precursor to the $41.5-\mathrm{kDa}$ pro-enzyme and a newly detected 41-kDa peptide. Eur. J. Biochem. 203, 641-653.

Johnson, L.M., Bankaitis, V.A., and Emr, S.D. (1987). Distinct sequence determinants direct intracellular sorting and modification of a yeast vacuolar proteinase. Cell 48, 875-885.

Kane, P.M., Kuehn, M.C., Howald, I., and Stevens, T.H. (1992). Assembly and targeting of peripheral and integral membrane subunits of the yeast vacuolar $\mathrm{H}^{+}$-ATPase. J. Biol. Chem. 267, 447-454.

Kane, P.M., Yamashiro, C.T., and Stevens, T.H. (1989). Biochemical characterization of the yeast vacuolar $\mathrm{H}^{+}$-ATPase. J. Biol. Chem. 26, 19236-19244.

Kaneko, Y., Hayashi, N., Toh-e, A., Banno, I., and Oshima, Y. (1987). Structural characteristics of the PHO8 gene encoding repressible alkaline phosphatase in Saccharomyces cerevisiae. Gene 58, 137-148.

Klionsky, D.J., Banta, L.M., and Emr, S.D. (1988). Intracellular protein sorting and processing of a yeast vacuolar hydrolase: proteinase $\mathrm{A}$ propeptide contains vacuolar targetting information. Mol. Cell. Biol. 8, 2105-2116

Klionsky, D.J., and Emr, S.D. (1989). Membrane protein sorting in yeast: biosynthesis, transport and processing of yeast vacuolar alkaline phosphatase. EMBO J. 8, 2241-2250. 
Klionsky, D.J., Herman, P.K., and Emr, S.D. (1990). The fungal vacuole: compostion, function, and biogenesis. Microbiol. Rev. 54, 266-292.

Moehle, C.M., Dixon, C.K., and Jones, E.W. (1989). Processing pathway for protease B of Saccharomyces cerevisiae. J. Cell Biol. 108, 309-324.

Preston, R.A., Manolsen, M., Becherer, K., Weidenhammer, E., Kirkpatrick, D., Wright, R., and Jones, E.W. (1991). Isolation and characterization of PEP3, a gene required for vacuolar biogenesis in Saccharomyces cerevisiae. Mol. Cell. Biol. 11, 5801-5812.

Pringle, J.R., Preston, R.A., Adams, A.E.M., Stearns, T., Drubin, D.G., Haarer, B.K., and Jones, E.W. (1989). Fluorescence microscopy methods for yeast. Methods Cell Biol. 31, 357-435.

Raymond, C.K. (1990). Molecular analysis of genes required for vacuolar protein sorting in the yeast Saccharomyces cerevisiae. $\mathrm{PhD}$ thesis. Eugene, OR: University of Oregon.

Raymond, C.K., O'Hara, P.J., Eichinger, G., Rothman, J.H., and Stevens, T.H. (1990). Molecular analysis of the yeast VPS3 gene and the role of its product in vacuolar protein sorting and vacuolar segregation during the cell cycle. J. Cell Biol. 111, 877-892.

Raymond, C.K., Roberts, C.J., Moore, K.E., Howald-Stevenson, I., and Stevens, T.H. (1992a). Biogenesis of the vacuole in Saccharomyces cerevisiae. Int. Rev. Cytol. 139, 59-120.

Raymond, C.K., Vater, C.A., Nothwehr, S.F., Roberts, C.J., and Stevens, T.H. (1992b). The sorting of soluble and integral membrane proteins to the yeast vacuole. In: Membrane Biogenesis and Protein Targeting, ed. W. Neupert, and R. Lill, Cambridge, UK. Elsevier Publishing, Inc., 165-182.

Redding, K., Holcomb, C., and Fuller, R.S. (1991). Immunolocalization of Kex2 protease identifies a putative late Golgi compartment in the yeast Saccharomyces cerevisiae. J. Cell Biol. 113, 527-538.

Roberts, C.J. (1991). Membrane protein sorting in the secretory pathway of Saccharomyces cerevisiae. PhD. thesis. Eugene, OR: University of Oregon.

Roberts, C.J., Nothwehr, S.F., and Stevens, T.H. (1992). Membrane protein sorting in the yeast secretory pathway: evidence that the vacuole may be the default compartment. J. Cell Biol. 119, 69-83.

Roberts, C.J., Pohlig, G., Rothman, J.H., and Stevens, T.H. (1989). Structure, biosynthesis, and localization of dipeptydyl aminopeptidase $B$, an integral membrane glycoprotein of the yeast vacuole. J. Cell Biol. 108, 1363-1373.

Roberts, C.J., Raymond, C.K., Yamashiro, C.T., and Stevens, T.H. (1991). Methods for the study of the yeast vacuole. Methods Enzymol. 194, 644-661.

Robinson, J.S., Graham, T.R., and Emr, S.D. (1991). A putative zinc finger protein, Saccharomyces cerevisiae Vps18p, affects the late Golgi functions required for vacuolar protein sorting and efficient $\alpha$-factor prohormone maturation. Mol. Cell. Biol. 11, 5813-5824.

Robinson, J.S., Klionsky, D.J., Banta, L.M., and Emr, S.D. (1988). Protein sorting in Saccharomyces cerevisiae: isolation of mutants defective in the delivery and processing of multiple vacuolar hydrolases. Mol. Cell. Biol. 8, 4936-4948.

Rothman, J.H., Howald, I., and Stevens, T.H. (1989a). Characterization of genes required for protein sorting and vacuolar function in the yeast Saccharomyces cerevisiae. EMBO J. 8, 2057-2065.

Rothman, J.H., Hunter, C.P., Valls, L.A., and Stevens, T.H. (1986). Overproduction-induced mislocalization of a yeast vacuolar protein allows isolation of its structural gene. Proc. Natl. Acad. Sci. USA 83, 3248-3252.

Rothman, J.H., Raymond, C.K., Gilbert, T., O'Hara, P.J., and Stevens, T.H. (1990). A putative GTP-binding protein homologous to interferon-inducible $\mathrm{Mx}$ proteins performs an essential function in yeast protein sorting. Cell 61, 1063-1074.
Rothman, J.H., and Stevens, T.H. (1986). Protein sorting in yeast: mutants defective in vacuole biogenesis mislocalize vacuolar proteins into the late secretory pathway. Cell 47, 1041-1051.

Rothman, J.H., Yamashiro, C.T., Raymond, C.K., Kane, P.M., and Stevens, T.H. (1989b). Acidification of the lysosome-like vacuole and the vacuolar $\mathrm{H}^{+}$-ATPase are deficient in two yeast mutants that fail to sort vacuolar proteins. J. Cell Biol. 109, 93-100.

Shaw, J.M., and Wickner, W. (1991). vac2: a yeast mutant which distinguishes vacuole segregation from Golgi-to-vacuole protein targeting. EMBO J. 10, 1741-1748.

Sherman, F., Fink, G.R., and Hicks, J.B. (1982). Methods in Yeast Genetics, Cold Spring Harbor, New York: Cold Spring Harbor Laboratory.

Singer, B., and Riezman, H. (1990). Detection of an intermediate compartment involved in transport of $\alpha$-factor from the plasma membrane to the vacuole in yeast. J. Cell Biol. 110, 1911-1922.

Stevens, T.H., Esmon, B., and Schekman, R. (1982). Early stages in the yeast seceretory pathway are required for the transport of carboxypeptidase $Y$ to the vacuole. Cell 30, 439-448.

Stevens, T.H., Rothman, J.H., Payne, G.S., and Schekman, R. (1986). Gene dosage-dependent secretion of yeast vacuolar carboxypeptidase Y. J. Cell Biol. 102, 1551-1557.

Valls, L.A., Hunter, C.P., Rothman, J.H., and Stevens, T.H. (1987). Protein sorting in yeast: the localization determinant of yeast carboxypeptidase $Y$ resides in the propeptide. Cell 48, 887-897.

Valls, L.A., Winther, J.R., and Stevens, T.H. (1990). Yeast carboxypeptidase $Y$ vacuolar targetting signal is defined by four propeptide amino acids. J. Cell Biol. 111, 361-368.

Wada, Y., Kitamoto, K., Kanbe, T., Tanaka, K., and Anraku, Y. (1990). The SLP1 gene of Saccharomyces cerevisiae is essential for vacuolar morphogenesis and function. Mol. Cell. Biol. 10, 2214-2223.

Weimken, A., Matile, P., and Moor, H. (1970). Vacuolar dynamics in synchornously budding yeast. Arch. Mikrobiol. 70, 89-103.

Weisman, L.S., and Wickner, W. (1988). Intervacuolar exchange in the yeast zygote: a new pathway in organelle communication. Science $241,589-591$.

Weisman, L.S., and Wickner, W. (1992). Molecular characterization of VAC1, a gene required for vacuole inheritance and vacuolar protein sorting. J. Biol. Chem. 267, 618-623.

Weisman, L.S., Bacallao, R., and Wickner, W. (1987). Multiple methods of visualizing the yeast vacuole permit evaluation of its morphology and inheritance during the cell cycle. J. Cell Biol. 105, 1539-1547.

Weisman, L.S., Emr, S.D., and Wickner, W. (1990). Mutants of Saccharomyces cerevisiae that block intervacuole vesicular traffic and vacuole division and segregation. Proc. Natl. Acad. Sci. USA 87, 1076-1080.

Wilcox, C.A., Redding, K., Wright, R., and Fuller, R.S. (1992). Mutation of a tyrosine localization signal in the cytosolic tail of yeast Kex2 protease disrupts Golgi retention and results in default transport to the vacuole. Mol. Biol. Cell 3, 1353-1371.

Woolford, C.A., Dixon, C.K., Manolson, M.F., Wright, R., and Jones, E.W. (1990). Isolation and characterization of PEP5, a gene essential for vacuolar biogenesis in Saccharomyces cerevisiae. Genetics 125, 739-752.

Yamashiro, C.T. (1990). A biochemical and genetic analysis of the yeast vacuolar proton-translocating adenosine triphosphatase, PhD thesis. Eugene, OR: University of Oregon.

Yamashiro, C.T., Kane, P.M., Wolczyk, D.F., Preston, R.A., and Stevens, T.H. (1990). The role of vacuolar acidification in protein sorting and zymogen activation: a genetic analysis of the yeast vacuolar $\mathrm{H}^{+}-$ ATPase. Mol. Cell. Biol. 10, 3737-3749.

Zubenko, G.S., Park, F.J., and Jones, E.W. (1982). Genetic properties of mutations at the PEP4 locus in Saccharomyces cerevisiae. Genetics. 102, 679-690. 